\title{
Study on Characteristics of Exercise Load of Football Referees on the Spot
}

\author{
Xuanwu Zhang ${ }^{1, *}$ \\ ${ }^{1}$ Guangzhou Vocational and Technical University of Science and Technology, Guangzhou, Guangdong 510450, China \\ *Corresponding author.
}

\begin{abstract}
In this study, by collecting the data of running distance, heart rate index and rating of perceived exertion (RPE) index of China's national and first-level chief referees in different competitions, the load difference of chief referees in officiating competitions was scientifically quantified. The chief referee of football was taken as research object, including 6 first-class referees registered by Chinese Football Association and 12 national referees. The experimental test method was applied in the study to monitor the internal and external loads of the referee wearing Polar heart rate band and Catapult OptimEyeS5 equipment during competition, and give the subjective fatigue scale of the referee measured by rating of perceived exertion (RPE) scale before and after the match. Statistical Product and Service Solutions (SPSS) software was employed for statistical analysis of the data collected through experimental tests: The independent sample $T$ test was conducted for the mean values of all running distances of the first level referees and the national referees in the provincial governor cup and the national referees in the provincial governor cup and the U20 under different running forms (sprint, high speed, medium speed, low speed, jogging). The independent sample $T$ test was performed for the mean distribution proportion of the maximum heart rate (HR Max) interval (low intensity heart rate, medium and low intensity heart rate, medium intensity heart rate, medium and high intensity heart rate, and high intensity heart rate). The mean value of running distance of all fields and the subjective fatigue value of different acceleration interval were tested by the independent sample $T$ test, and the significant difference of running distance between the whole field and the half court was analyzed. Paired sample $T$ was used to test whether there were any differences in the indexes of the second half of the same game. Results show that there was no difference in the exercise load of the chief referee in the first and second half of the judging process. The national umpire's exercise load at the U20 is greater than that at the governor cup. The running distance of the first-class judges is $\mathbf{3 4 4}$ meters higher than that of the national judges in low intensity running, and 193 meters higher than that of the national referees in medium intensity running. The main difference centered on the second half. The proportion of lowintensity heart rate of national referees reached $\mathbf{7 3 . 4 \%}$, while that of first-class referees reached $58.8 \%$. The running distance of second-half low-intensity acceleration of first-class referees was $\mathbf{2 , 4 4 0}$ meters, higher than that of national referees. When the national referee and the first-class judge adjudicate the same competition, the sports load of the former is lower than the latter. When they are in the U20 competition, the total running distance reached 8,910 meters, higher than that in governor cup, the running distance of low intensity was 699 meters, the running distance of medium intensity was 398 meters, and the running distance of high intensity was 208 meters. The running amount and load were both higher than that in governor cup. During the U20 competition, the average heart rate was 161.4 times/min, the maximum heart rate was $189.5 \mathrm{times} / \mathrm{min}$ in the second half, the proportion of lowintensity heart rate was $\mathbf{2 7 . 0 \%}$, and the proportion of low-intensity heart rate was $\mathbf{7 3 . 2 \%}$, both higher than that of governor cup. The running distance of low-intensity acceleration reached 5,113 meters, higher than that of the governor cup.
\end{abstract}

Keywords: exercise load, running form, heart rate, acceleration, referee 


\section{INTRODUCTION}

With the development of football as a national development strategy, the popularity of football is becoming higher and higher, and the development of grassroots referees is urgent. The tempo of offense and defense in modern football matches is getting faster and faster, and the athletic has reached a high level. The improvement of football tactics has promoted the players to make a qualitative leap in running distance, which marks the further development of the speed of the football game, and the level of confrontation, and higher requirements on referee's physics, indicating that the cultivation and training of referees shall be more systematic and scientific.

\section{A. Research significance and purpose}

It is necessary to carry out systematic and scientific training for referees in terms of physical ability, eliminate empirical referee training, quantify the referee's workload in youth football matches, and formulate specific sports training plans based on the required sports load, which has certain scientific basis for training the reserve force of football referee.

Its theoretical significance lies in that the improvement of the comprehensive professional ability of football referees is always a problem in football matches. Starting from the construction of the reserve force team of football referees, it is especially important to emphasize and cultivate their comprehensive ability. The theoretical knowledge of sports training of football referees should be enriched to provide theoretical basis for the better development of football in China.

Its significance in practical application are as follows: through quantifying the workload of the referee on the spot, the running characteristics and heart rate characteristics of the first and national referees were compared and analyzed. The running forms in the field judgment, such as sprint, high speed running proportion, and the proportion of $80 \%$ to $90 \%$ of the maximum heart rate in the field judgment, were studied. Then the referees are guided to develop a specific exercise plan, and scientific, systematic, and reasonable advice is proposed to carry out more effective physical training.

\section{B. Literature review}

1) Definition of concept: Exercise load: the exercise load refers to the total stimulation that the body bears in physiology and psychology when the athlete is subjected to certain external stimulation. [1] Exercise load falls into two camps: external and internal load. The former can be evaluated by such methods as running distance and running speed of football players, while the latter can be monitored by heart rate, oxygen consumption and rating of perceived exertion scale (RPE). [2]

Heart rate: heart rate refers to the number of heart beats per minute in a normal person's quiet state, also known as resting heart rate. The overall load of the game is the load borne by the athletes in a game. Since the two systems of body consumption and recovery work simultaneously in the game, it reflects the immediate state of the athletes' body after a complete game, and can be evaluated according to the physiological indicators immediately after the end of the game. The most common indicators are heart rate and blood lactic acid, and heart rate is the most appropriate from the operational point of view. [3] [4] In this paper, the equipment employed to test the chief referee of football matches is Polar heart rate band, which has been widely used in sports load-related studies.

Exercise load in football match: It is the stimulation of a player's body by various physical activities during a football match. Due to the limitation of competition rules, it is difficult to evaluate the physiological changes of athletes in time, and the characteristics of the changes cannot be accurately analyzed.

Running ability: it refers to the athletes' ability to carry out various forms of activities in the competition, which is directly reflected by running distance. Running distance: it refers to the sum of the distance that the athlete moves when he or she is engaged in various activities during the race. [5]

RPE: It refers to the subjective evaluation made by individuals of their overall feelings of fatigue during intense exercise. In the modified 15-grade RPE rating, Borg, the founder, connected the subjective sensation during exercise with the pulse and heartbeat during exercise, and calculated HR=RPEx10, that is, the heart rate of participants during exercise is equal to RPE series multiplied by 10 . Despite the fact that heart rate is affected by environmental, psychological, exercise patterns and other factors, Borg's assessment has achieved a high reliability coefficient over the years. [6] Iilmpellizzeri (2012) summarized in Use of Session RPE to Training Load Quantification and Training Intensity Distribution in Taekwondo Athletes that RPE can be adopted to monitor the quality of daily training, and is conducive to heart rate monitoring and some basic research of biotechnology. [7]

2) Study on college students and professional football players: Yang Zeyi et al. (2004) wrote in Physical Fitness and Nutrition in Football that football is a sport that requires a high level of physical fitness. It belongs to one of the few that require both speed and agility, as well as strength, explosive power and endurance. [8] Tian Zhiqi and Sun Bing (2007) pointed out that there is a significant difference in physical 
fitness and some functions between high-level male football players and professional football players in ordinary colleges and universities in Tianjin, mainly in the $12 \mathrm{~min}$ run, explosive power test, standing leap, shuttle run and sprint ability. [9] Li Qiang et al. (2014) recorded 21 National Olympic players (China U23 national football team) and 25 national youth players (China U19 national football team) when researching and analyzing the characteristics of high-intensity running changes of outstanding young football players. The performance and physical fitness data of the 3 to 6 competitions participated in the season show that the distance of the high-intensity running of the Olympic team athletes in the game is significantly longer than that of the national youth team. [10] The high-intensity running here refers to medium-speed running, highspeed running, and sprint running. [11] Hu Xin (2015) believes that college football players perform poorly due to the gap in practice time and intensity between them and professional athletes. [12]

There is a certain gap between the physical quality and running of college football players and professional sports, and with the growth of the age of football players, their high-intensity running in the game is getting higher and higher, which shows that the level of the game is related to the age of players.

3) Research on running ability against external load: In Characteristics of Running Distance in the Match of China National Men's Football Players, Zhu Junkai and Liu Dan take the running distance of Chinese and foreign football players of 106 persontimes in 12 matches in 2004 Asian Cup, 2008 EAFF East Asian Cup, 2009 China VS Senegal, 2010 China VS Iran, China VS Paraguay as the research objects, and divided the running form and speed into the following camps: sprint with speed $\geq 8.3 \mathrm{~m} / \mathrm{s}$; highspeed running with speed $\geq 5.8 \mathrm{~m} / \mathrm{s}$; medium-speed running with speed $\geq 4.4 \mathrm{~m} / \mathrm{s}$; low-speed running with speed $\geq 3.3 \mathrm{~m} / \mathrm{s}$; jogging with speed $\geq 2.2 \mathrm{~m} / \mathrm{s}$; and the speed of other activities is $<2.2 \mathrm{~m} / \mathrm{s}$. It is concluded that except for the goalkeeper, the distance and total distance of the athletes at all levels of running speed in the second half are less than that in the first half. In the second half, the distance of high intensity activity decreased significantly. [13]

In Relationship between Physical Performance and Offensive Effect for Elite Woman Soccer Players, Bu Yifeng (2014) took 30 national team matches as the research object. Sprint $\geq 6 \mathrm{~m} / \mathrm{s}$ in high-intensity running is anaerobic running mainly powered by ATP/CP; High-speed running $\geq 4.8 / \mathrm{s}$ is mainly provided by anaerobic fermentation. Medium-speed running $\geq 3.6 / \mathrm{s}$ in medium-intensity running is dominated by anaerobic fermentation. In low-intensity running, low-speed running $\geq 2.4 \mathrm{~m} / \mathrm{s}$ is aerobic and belongs to aerobic running. Jogging $\geq 1.5 \mathrm{~m} / \mathrm{s}$ is mainly provided with aerobic energy, and other running activities $<1.5 \mathrm{~m} / \mathrm{s}$. [14]

Zhang Yingcheng, and Chen Gang (2014) et al. studied the characteristics of high-level women's soccer referees' game behaviors and activities, using the revised running speed rating standard for women by $\mathrm{Bu}$ Yifeng. [15]

Li Xiaokang et al. (2016) divided running speed according to UEFA standards: walking: $\mathrm{V}>0 \leq$ $11 \mathrm{KM} / \mathrm{H}$; jogging: $\mathrm{V}>11 \mathrm{KM} / \mathrm{H} \leq 14 \mathrm{KM} / \mathrm{H}$; running: $\mathrm{V}>14 \mathrm{KM} / \mathrm{H} \leq 17 \mathrm{KM} / \mathrm{H}$; fast running: $\mathrm{V}>17 \mathrm{KM} / \mathrm{H} \leq$ $21 \mathrm{KM} / \mathrm{H}$; high-speed running: $\mathrm{V}>21 \mathrm{KM} / \mathrm{H} \leq$ $24 \mathrm{KM} / \mathrm{H}$; and sprint: $\mathrm{V}>24 \mathrm{KM} / \mathrm{H}$. [16]

$\mathrm{Yu}$ Shaohua et al. (2009) adopted the standard developed by Bangsbo, the Danish scholar who carried out the study, to divide different running speeds: anaerobic high-intensity running includes sprint (speed $\geq 8.3 \mathrm{~m} / \mathrm{s}$ ) and high-speed running (speed $\geq 5.8 \mathrm{~m} / \mathrm{s}$ ). Aerobic moderate-intensity running includes: mediumspeed running (speed $\geq 4.4 \mathrm{~m} / \mathrm{s}$ ), low-speed running (speed $\geq 3.3 \mathrm{~m} / \mathrm{s}$ ). Aerobic low-intensity running includes: jogging (speed $\geq 2.2 \mathrm{~m} / \mathrm{s}$ ), other activities slower than jogging (speed $<2.2 \mathrm{~m} / \mathrm{s}$ ). [17]

In his work, Bangsbo (1994) conducted a systematic study of the distance of movement of European athletes. Liu Dan, citing the running distance characteristics of European athletes analyzed by Bangsbo, concluded in the article Study on Running Ability of Chinese Men Excellent Football Players during Match that, compared with the European athletes, the athletes of the Chinese national team have a significant difference in the distance of activities, which is mainly reflected in the distance of sprints and low-speed running. In terms of running distance and high-intensity running distance in various periods, Chinese athletes' ability of repeated sprints in the competition, as well as their ability to eliminate lactic acid and provide energy with moderate-intensity aerobic running, is obviously different from that of European athletes. [18] [19]

In conclusion, the athletes' speed is the embodiment of their activity form. In this study, the running form and speed of the test objects are divided into the following: the sprint speed is greater than $24 \mathrm{~km} / \mathrm{h}$, the speed of high-speed running is $21 \mathrm{~km} / \mathrm{h}-23.9 \mathrm{~km} / \mathrm{h}$, of medium-speed running is $17 \mathrm{~km} / \mathrm{h}-20.9 \mathrm{~km} / \mathrm{h}$, of lowspeed running is $14 \mathrm{~km} / \mathrm{h}-16.9 \mathrm{~km} / \mathrm{h}$, of jogging is $11 \mathrm{~km} / \mathrm{h}-13.9 \mathrm{~km} / \mathrm{h}$, and the walking speed is less than $11 \mathrm{~km} / \mathrm{h}$.

4) Study on strength interval of heart rate among internal load: Australia's Picher points out in Pulse Rate - A Sign of Intensity During Training on 
swimming training that there is a significant correlation between the daily morning pulse and the pulse during activities and his exercise intensity and competition results. The use of the recorded pulse frequency system to determine the biological cycle of training can enable the coach to know exactly how much exercise to complete, when to train and how much intensity. In the article Training for Finnish Long Distance Runners, Cinconen from Finland pinpoints that running a certain distance with a heart rate of 140-170 times/min was the criterion to evaluate the intensity of training. In the article Heart Rate and Training Intensity, Kaga Taniko points out: Heart rate is one of the easiest and most reliable measures of the cardiovascular system. There is a linear relationship between heart rate and physical load intensity, heart rate and oxygen uptake (i.e. proportional). [20]

Deng Weiming et al. (2004) proposed in their research on physiological load in physical strength training that the intensity level of the internal load is divided into 10 grades according to the percentage of the maximum physiological load that the athletes can achieve. Such ranges as $\leq 40 \%, 40 \%-49 \%, 50 \%-59 \%$, $60-69 \%, 70 \%-79 \%, 80 \%-89 \%$, and $90 \%-100 \%$ are represented by PWI Max. [21]

Dong Fangyu (2016) used the American Sports Medical Association's exercise intensity distribution table to study the load characteristics of Chinese men's international referees in the field. The ultimate strength is $>90 \% \mathrm{HR}$ max, the high strength is $70 \%$ $89 \%$ HRmax, the medium strength is $55-69 \%$ HR max, and the low strength is $35 \%-54 \%$ HR max. [22]

Chen Chao and Liu Dan (2010) analyzed in the article Study on Features of the Heart Rate of Chinese Women Football Team Players during Match and pointed out that the heart rate during match was adopted to evaluate the load, and athletes' heart rate were divided into 4 intervals: $<75 \%$ represents the maximum heart rate, $75 \% \sim 85 \%$ the maximum heart rate, $85 \% \sim 90 \%$ the maximum heart rate, and $>90 \%$ the maximum heart rate. It is concluded that the average load intensity and high intensity exercise time of Chinese women's national team athletes in the first half are higher than that in the second half. [23]

The maximum heart rate (HR max) is the maximum number of times per minute the heart can contract. The maximum heart rate decreases with the increase of age. The maximum heart rate of an individual can be estimated according to the formula "maximum heart rate $=220$ - age". The maximum heart rate was independent of physical fitness and exercise training. [24]

To sum up, heart rate is the main monitoring index of internal load in this study, and the intensity interval divided by heart rate is different among scholars. In this study, the intensity intervals of each heart rate were divided into the following intensity intervals according to the intensity of physiological load response and with reference to previous studies: the low intensity heart rate is less than $60 \% \mathrm{HR}$ max, medium and low intensity heart rate is $60-69 \% \mathrm{HR}$ max, medium intensity heart rate is $70-79 \% \mathrm{HR}$ max, medium and high intensity heart rate is $80 \%-89 \%$ HR max, high intensity heart rate is $90 \%-100 \%$ HR max, etc.

5) Study on the division of motion acceleration and intensity interval: In China, there is almost no interval division and definition of athletes' speed in sports. In this paper, the motion acceleration of the referee in the process of judging is divided into 8 intervals (not defined): high-speed deceleration running, acceleration: minus 20 - minus $4 \mathrm{~m} / \mathrm{s}^{2}$; moderate and high intensity deceleration running, acceleration: minus 4 - minus $3 \mathrm{~m} / \mathrm{s}^{2}$; moderate-intensity deceleration running, acceleration: minus 3 - minus $2 \mathrm{~m} / \mathrm{s}^{2}$; low-intensity deceleration running, acceleration: $-2-0 \mathrm{~m} / \mathrm{s}^{2}$; lowintensity accelerated running, acceleration: $0-2 \mathrm{~m} / \mathrm{s}^{2}$; medium-speed running, acceleration: $2-3 \mathrm{~m} / \mathrm{s}^{2}$; mediumhigh speed running, acceleration: $3-4 \mathrm{~m} / \mathrm{s}^{2}$; and highspeed running, acceleration: $4-20 \mathrm{~m} / \mathrm{s}^{2}$.

6) Evaluation and application of the RPE scale to the sports load in football matches: Peng Li, Cao Zeliang et al. (2011) analyzed whether the heart rate estimated by Borg's formula HR= RPEx10 was reliable against different exercise load schemes for men. It is verified that whether the RPE value is highly correlated with the heart rate provides some basis for improving the effectiveness and objectivity of RPE evaluation. The difference between the estimated rate of constant load motion and the actual value is lower than that between the estimated rate of increasing load motion and the actual value, so the estimated effect should be more effective. [25]

Wu Jingcheng et al. (2011) studied the subjects who performed incremental motion to exhaustion on the power bicycle. They were asked about their RPE in the last 20 seconds of each level of load, and concluded that the actual breathing rate might be more effective in evaluating RPE value.

Liu Hongyou (2015) argues that RPE is a practical, simple and cheap way to monitor the internal load, and Application of a Modified Rating of Perceived Exertion Scale and Weight Loss on the Control of Football Training Load studies the improved 10-level RPE can be employed to collect the "perceived exertion rating before training" and "perceived exertion rating after training" of athletes, so as to obtain "cumulative perceived exertion scale in training". [26] (See "Fig. 1") 


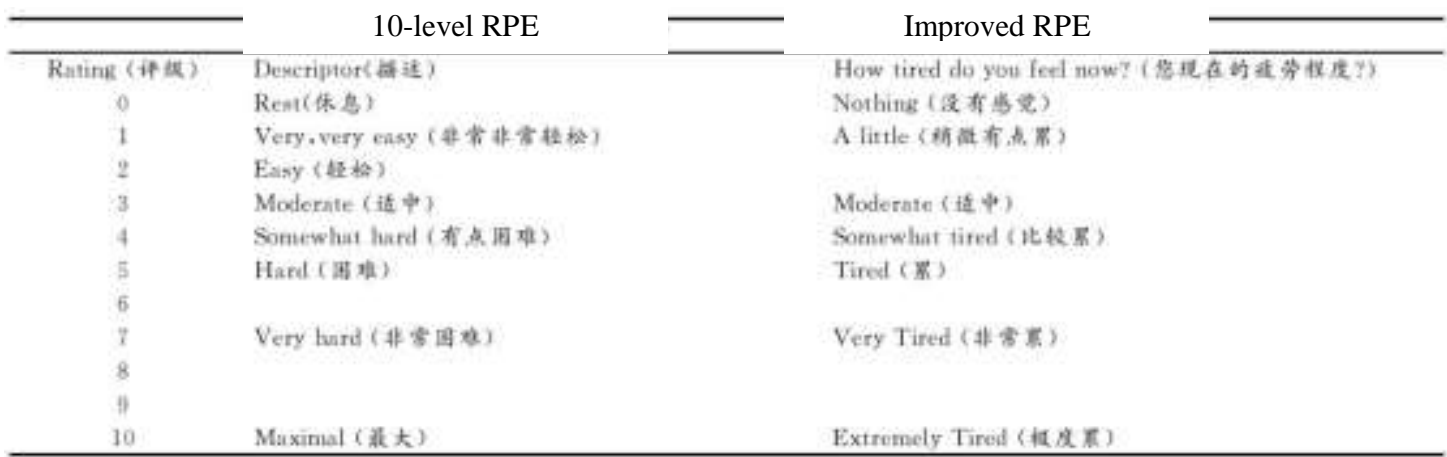

Fig. 1. RPE.

The research results of Impellizzeri $\mathrm{F}$ M. et al. (2004) shows that RPE enjoys a correlation in the monitoring of football training load [27], and a certain correlation in the use of RPE in the training load quality of taekwondo athletes (2012). [28]

To sum up, this study evaluated the overall exercise load of the referee on the spot through the improved 10level RPE.

7) Use of test equipment: Regarding the application of Polar heart rate band, Liu Panpan (2016) proposed in Thinking about Preventing Sudden Death in Marathon Based on Polar Heart Rate Data that as an aerobic project, marathon can be effectively monitored by Polar heart rate watch and heart rate band during training and competition. [29] Wang Benjie (2010) held in An Analysis System of Physical Training Workload Based on Polar Sports Tester that the Polar watch with Finland is one of the most common instruments in sports training, and it plays a very important role in monitoring the training intensity of athletes.

\section{RESEARCH OBJECTS AND METHODS}

\section{A. Research objects and main content}

The research object of this paper is football referees. The author tests referees who are registered with Chinese Football Association and already have the experience of judging matches held by Chinese Football Association. Based on the governor cup and the U20 series invitational tournament, this paper analyzes the sports load of the football referees with the first-class and national qualifications, and quantifies the running form, distance, and heart rate characteristics.

The referee's external load during the on-site ruling is assessed by his ability to run on the field. In this study, the total running distance and running distance under each speed range of the referee at different levels were tested through experiments, and the differences of external load characteristics of the first-class and national referees were analyzed. Internal load is mainly assessed by heart rate. RPE is a practical, simple and inexpensive method for monitoring exercise load. The RPE of the chief referee was identified 30 minutes before and after the race, and the differences in the comprehensive load characteristics of the first-class and national referees were analyzed.

\section{B. Research methods}

1) The method of literature: This paper, with the aid of library of South China Normal University, CNKI, Wanfang Data, and network, searches related journals and master's thesis with football referees, exercise load, running distance, heart rate, and referee grade as keywords, classifies literature, and analyzes representative and authoritative literature to deepen understanding.

\section{2) Experimental test method}

a) Test object: This paper selects referees who are registered in Chinese Football Association and have experience in holding matches of Chinese Football Association, including 6 first-class referees and 12 national referees. They were tested for exercise load in the international invitational match of officiating on 610 November 2017 and the provincial cup match of officiating on November and December 2017, and the perceived exertion was determined before and after the match. A total of 18 matches were tested, including 6 matches attended by first-class referees and 12 matches attended by national referees. Among them, there are 6 matches in the U20 series of the international invitational tournament. The opposing teams are respectively Guangzhou Evergrande, Guangzhou Fuli FC, Grêmio Football Porto Alegrense, and Brazil Santos. There are 12 matches in the provincial governor cup, and the teams are universities in Guangdong.

b) Test methods: Xu Zhibiao (2016) proposed in Practical Research on Evaluation of Exercise Intensity Using Polar Heart Rate Test Table in Physical Education that Polar heart rate table could be widely used in physical education, as it could provide simple, 
scientific, accurate and timely feedback of heart rate information. [30] Wen Xu (2017) proposed in Application of Smart Wearable Devices in the Big Data Analysis of Physical Activity in China that the data provided by smart wearable systems already have volume, velocity and variety, namely the $3 \mathrm{~V}$ characteristics of big data. Some smart wearable devices also have excellent economy and ease of use. It is necessary to assist the referee in testing Polar heart rate band and Catapult OptimEyeS5 equipment prior to the start of warm-up, and record the start and end times of warm-up and game. Ten minutes before the race, the RPE was given to evaluate the fatigue scale, and 30 minutes after the race, the fatigue value was evaluated again.

c) Test time: October 2017 to December 2017.

d) Test location: The test locations were Zhuhai Sports Center in Zhuhai City, Guangdong Province, Evergrande Football School in Qingyuan City, Guangdong Province, and universities in Guangzhou City, Guangdong Province.

3) Mathematical statistics: Football matches usually have stoppage time, which is more than 90 minutes and is calculated by the referee according to the injury or substitution of players on the field. Since the data of the statistical records of experimental tests are accompanied by supplementary time, this paper standardizes the data so that the sample data are 90 minutes. The specific operations are as follows: 90/specific length $*$ actual running distance $=$ total running distance of 90 minutes. Each index was standardized and analyzed by SPSS software. In the following statements, the first referee group A shall describe the first referee presiding over the provincial cup, the national referee group A shall describe the national referee presiding over the provincial cup, and the national referee group B shall describe the national referee presiding over the international invitational tournament.

An independent sample $\mathrm{T}$ test was conducted on the mean values of all running distances in different running forms (sprint, high speed, medium speed, low speed, jogging, and slower activities) between first referee group A and national referee group A to analyze whether there was a significant difference in running distances between the first and second half. The mean proportions of the distribution of the HR max interval (low-intensity heart rate, low and medium intensity heart rate, medium-intensity heart rate, medium and high intensity heart rate, high-intensity heart rate) in first referee group A and national referee group A were tested by independent sample $\mathrm{T}$ test to analyze whether there was a significant difference between the whole court and the first and second half. An independent sample $\mathrm{T}$ test was conducted on the mean running distance of the acceleration interval (high-intensity deceleration running, medium-high intensity deceleration running, medium-intensity deceleration running, low-intensity deceleration running, lowintensity acceleration running, medium-intensity acceleration running, medium-high intensity acceleration running, and high-intensity acceleration running) between first referee group $\mathrm{A}$ and national referee group A to analyze whether there was a significant difference between the whole court and the first and second half.

Independent sample $\mathrm{T}$ test was conducted on the mean values of all running distances in different running forms (sprint, high speed, medium speed, low speed, jogging, and slower activities) of national referee group A and group B. The mean of the proportion of the interval of HR max (low-intensity heart rate, low and medium intensity heart rate, medium-intensity heart rate, medium and high intensity heart rate, highintensity heart rate) was tested by the independent sample $\mathrm{T}$ test, and the mean of the running distance of all the fields in different acceleration intervals (highintensity deceleration running, medium-high intensity deceleration running, medium-intensity deceleration running, low-intensity deceleration running, lowintensity acceleration running, medium-intensity acceleration running, medium-high intensity acceleration running, and high-intensity acceleration running) was tested by the independent sample $\mathrm{T}$ test to analyze whether there is a significant difference in running distance between the whole court and the first and second half.

The paired sample $\mathrm{T}$ test was conducted on the first referee group A, national referee group A and national referee group B to analyze whether there was a significant difference in the whole court and the first and second half.

Independent sample $\mathrm{T}$ test was conducted for fatigue scale of national referees and first-class referees for governor cup, and of national referees for governor cup and U20 matches.

\section{CONCLUSION}

There was no difference in the total running amount of the referees in the 12 provincial cup, but there was a significant difference in the load. The exercise load of the first-class referees was higher than that of the national referees. The total distance of the first-class referee in the governor cup reaches 8,460 meters, which was not significantly different from that of the national referee. The running distance of the first-class referee was 344 meters for low-intensity running and 193 meters for medium-intensity running, both of which were higher than the national referees, and the main difference was reflected in the second half of the game. 
The proportion of low-intensity heart rate of national referees reached $73.4 \%$, while the proportion of medium and low intensity heart rate of first-class referees reached $58.8 \%$. The running distance of lowintensity acceleration running by first-class referees in the second half was 2,440 meters, higher than that of national referees. The reason is that the first-class referees are not as capable as national referee in judging competitions, resulting in high intensity running. The RPE results also confirmed the experimental results.

There are obvious differences in the total running distance and load of national referees when judging the U20 international invitational tournament and the provincial governor cup. Therefore, the higher the level of competition, the higher the exercise load of the referee of the same level. During the U20, the total running distance reached $8,910 \mathrm{~m}$, higher than the level in governor cup. The distance of low-intensity running is 699 meters, of medium-intensity running is 398 meters, and of high-intensity running is 208 meters. Running total and load are higher than the governor's cup. Running distance and load are both higher than that in governor cup. During the U20 competition, the average heart rate was 161.4 times/min, the HR max was 189.5 times/min in the second half, the proportion of low-intensity heart rate was $27.0 \%$, and the proportion of low-intensity heart rate was $73.2 \%$, both higher than that of governor cup. The low-intensity acceleration running reached 5,113 meters, higher than that of the governor cup. The RPE results also confirmed the experimental results.

Due to the small changes in the team during the match and the close rhythm of the match, there was no significant difference in the exercise load of the referee during the first and second half of the match.

\section{References}

[1] Tian Maijiu. Sports Training [M]. Beijing: Higher Education Press. 2006: 455. (in Chinese)

[2] Liu Hongyou, Jesus-Vicente Gimenez, Andres Alcolea Leon. Application of a Modified Rating of Perceived Exertion Scale and Weight Loss on the Control of Football Training Load [J]. China Sport Science. 2015-05-15: 1-2. (in Chinese)

[3] Zhao Gang, Liu Dan. Research on Constitution and Measurement of Physical Demand in Football Matches [J]. Journal of Capital College of Physical Education. 2013-05-15: 1-5. (in Chinese)

[4] Liu Dan. Football Physical Training: Theory and Practice of High-level football Physical Training [M]. Beijing: Beijing Sport University Press. 2006: 59-60. (in Chinese)

[5] Wu Jingcheng, Peng Li, Cao Zeliang, Yi Dongping. Research on Heart Rate and the Validity of Rating of Perceived Exertion under Different Exercise Load Plan [J]. China Sport Science and Technology. 2011-07-10: 1-2. (in Chinese)

[6] Impellizzeri F M. Use of session RPE to training load quantification and training intensity distribution in taekwondo athletes [J]. 2012.
[7] Yang Zeyi, Wang Qirong. Physical Fitness and Nutrition in Football [M]. Beijing: Beijing Sport University Press. 2004: 13. (in Chinese)

[8] Tian Zhiqi, Sun Bing. Study on the Constitution of High-level Male Soccer Players from Tianjin Colleges in Current China [J]. Journal of Nanjing Institute of Physical Education. 2007-04-15. (in Chinese)

[9] Li Qiang, Han Yuzong, De Hao. Variable Characteristics of High Intensity Running in Games of Chinese Elite Young Soccer Player $[\mathrm{J}]$. Journal of Tianjin Institute of Physical Education. 2014-03-25: 2-3. (in Chinese)

[10] Bangsbo J, Nørregaard L, Thors $\varnothing$ F. Activity profile of competition soccer $[\mathrm{J}]$. Canadian Journal of Sports Science, 1991, 16 (2)

[11] $\mathrm{Hu}$ Xin. The Effects on Aerobic Capacity of College Football Players with Two Aerobic Endurance Training Method [D] Xi'an Physical Education University. 2015-06-01: 3-4. (in Chinese)

[12] Zhu Junkai, Liu Dan. Analysis on Characteristics of Running Distance in the Match of China National Men's Football Players [J]. Journal of Beijing University of Physical Education. 201304-15: 2, 7. (in Chinese)

[13] Bu Yifeng. Relationship between Physical Performance and Offensive Effect for Elite Woman Soccer Players [J]. China Sport Science and Technology. 2014-03-15: 2; 7. (in Chinese)

[14] Zhang Yingcheng, Chen Gang, Bu Yifeng. Activity Profile and Physiological Demands of Top-class Woman Soccer Referees [J]. China Sport Science and Technology. 2014-09-15. (in Chinese)

[15] Li Xiaokang, Pan Chunguang, Liu Hao. Running Distance and Intensity Characteristics of Each Position Players in Chinese Football Association Super League [J]. Journal of Beijing University of Physical Education. 2016-03-15: 2. (in Chinese)

[16] Yu Shaohua, Liu Dan, Li Qiang. Study on Running Ability of Chinese Men Excellent Football Players during Match [J] China Sport Science and Technology. 2009-11-10: 1-2. (in Chinese)

[17] Bangsbo J. Energy Demands in Competitive Soccer [J]. JSports Sci, 1994, (12): 5-12.

[18] Bangsbo J. Fitness Traning in Football [M]. Copenhagen Krogh Institute University of Copenhagen Denmark, 1994

[19] Gao Daan, Meng Xianwu. Preliminary Discussion on the Heart Rate of Football Players in Matches and Training Centers [J]. China Sport Science and Technology. 1979-02-10: 2; 6. (in Chinese)

[20] Deng Weiming, Sun Xuechuan, Fan Xiaoyan. An Analysis System of Physical Training Workload Based on Polar Sports Tester [J]. Journal of Biomedical Engineering. 2004-10-30: 3-5. (in Chinese)

[21] Dong Fangyu. The Study of Chinese Male International Soccer Referees Loads Characteristics During the Official Matches [D]. Shanghai University of Sport. 2016-06-01: 16. (in Chinese)

[22] Chen Chao, Liu Dan, Fang Zhijun, Cao Xiaodong, Wang Xinluo. Study on Features of the Heart Rate of Chinese Women Football Team Players during Match [J]. China Sport Science. 2010-05-15. (in Chinese)

[23] Peng Li. Study on Maximum Heart Rate in Graded Exercise [J] China Sport Science and Technology. 2009-01-10: 1-5. (in Chinese)

[24] Wu Jingcheng, Peng Li, Cao Zeliang, Yi Dongping. Research on Heart Rate and the Validity of Rating of Perceived Exertion under Different Exercise Load Plan [J]. China Sport Science and Technology. 2011-07-10: 1-2. (in Chinese)

[25] Liu Hongyou, Jesus-Vicente Gimenez, Andres Alcolea Leon. Application of a Modified Rating of Perceived Exertion Scale 
and Weight Loss on the Control of Football Training Load [J]. China Sport Science. 2015-05-15: 1-2. (in Chinese)

[26] Impellizzeri F M. Rampinini E, coutts A J, et al. Use of RPEbased training load in soccer [J] Med Sci Sports Exe, 2004, 36 (6): 1042-1047.

[27] Mpellizzeri F M. Use of session RPE to training load quantification and training intensity distribution in taekwondo athletes [J]. 2012.

[28] Liu Panpan. Thinking about preventing sudden death in marathon based on Polar heart rate data [J]. Contemporary Sports Technology. 2016-07-05: 1-2. (in Chinese)

[29] Xu Zhibiao, Gu Yongming, Wang Ping, Zhu Bin. Practical Research on Evaluation of Exercise Intensity Using Polar Heart Rate Test Table in Physical Education. Teaching of Physical Education. 2016-08-15: 2. (in Chinese)

[30] Wen Xu, Yuan Bing, Li Hua, Zhou Houdong. Application of Smart Wearable Devices in the Big Data Analysis of Physical Activity in China [J]. China Sport Science and Technology. 2017-03-15: 4. (in Chinese) 